\title{
Article
}

\section{Surface assembly of cobalt species for simultaneous acceleration of interfacial charge separation and catalytic reactions on $\mathrm{Cd}_{0.9} \mathrm{Zn}_{0.1} \mathrm{~S}$ photocatalyst}

\author{
Khakemin Khan a,b, Lifen Xu a,b, Ming Shi a,b, Jiangshan Qu a,b, Xiaoping Tao a, Zhaochi Feng a, Can Li ${ }^{\text {a }}$ \\ Rengui Li ${ }^{a, *}$ \\ a State Key Laboratory of Catalysis, Dalian Institute of Chemical Physics, Chinese Academy of Sciences, Dalian National Laboratory for Clean Energy, \\ Dalian 116023, Liaoning, China \\ b University of Chinese Academy of Sciences, Beijing 100049, China
}

\section{A R T I C L E I N F}

\section{Article history:}

Received 24 August 2020

Accepted 11 September 2020

Available online 22 November 2020

\section{Keywords:}

Hierarchical heterostructure

Interfacial charge separation

Surface reaction

Photocatalytic hydrogen evolution

\begin{abstract}
A B S T R A C T
Although photocatalytic water splitting has excellent potential for converting solar energy into chemical energy, the challenging charge separation process and sluggish surface catalytic reactions significantly limit progress in solar energy conversion using semiconductor photocatalysts. Herein, we demonstrate a feasible strategy involving the surface assembly of cobalt oxide species $\left(\mathrm{CoO}_{x}\right)$ on

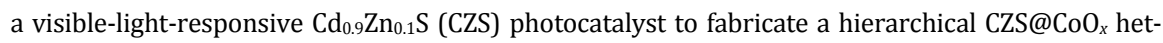
erostructure. The unique hierarchical structure effectively accelerates the directional transfer of photogenerated charges, reducing charge recombination through the smooth interfacial heterojunction between CZS and $\mathrm{CoO}_{x}$, as evidenced by photoluminescence (PL) spectroscopy and various electrochemical characterizations. The surface cobalt species on the CZS material also act as efficient cocatalysts for photocatalytic hydrogen production, with activity even higher than that of noble metals. The well-defined CZS@ $\mathrm{CoO}_{x}$ heterostructure not only enhances the interfacial separation of photoinduced charges, but also improves surface catalytic reactions. This leads to superior photocatalytic performances, with an apparent quantum efficiency of $20 \%$ at $420 \mathrm{~nm}$ for visible-light-driven hydrogen generation, which is one of the highest quantum efficiencies measured among noble-metal-free photocatalysts. Our work presents a potential pathway for controlling complex charge separation and catalytic reaction processes in photocatalysis, guiding the practical development of artificial photocatalysts for successful transformation of solar to chemical energy.
\end{abstract}

(C) 2021, Dalian Institute of Chemical Physics, Chinese Academy of Sciences. Published by Elsevier B.V. All rights reserved.

\section{Introduction}

Photocatalytic water splitting through particulate photocatalysts has gained considerable interest, owing to its promising applications for the conversion of earth-abundant resources into solar fuels [1-3]. As critical challenges and long-standing obstacles in photocatalytic water splitting, spatial separation and transport of photogenerated charges, together with the subsequent catalytic reactions, inevitably determine the energy conversion efficiency of artificial photocatalysts $[4,5]$. It is well

\footnotetext{
* Corresponding author. Tel: +86-411-84379698; Fax: +86-411-84694447; E-mail: rgli@dicp.ac.cn This work was financially supported by the National Natural Science Foundation of China (21633010, 21673230), Dalian Institute of Chemical Physics (DICP I202031), and Liaoning Revitalization Talents Program (XLYC1907078).

DOI: 10.1016/S1872-2067(20)63717-2 | http://www.sciencedirect.com/science/journal/18722067 | Chin. J. Catal., Vol. 42, No. 6, June 2021
} 
established that the recombination process of photogenerated charges can be prevented by using different approaches, including Schottky junctions [6], morphology engineering [7], phase junctions [8], and crystal facet engineering [9], in addition to surface modifications [10-12]. Moreover, depositing suitable cocatalysts enables the efficient trapping of photogenerated charges and provides reactive sites for the reduction and oxidation reactions, facilitating the utilization of photogenerated charges in photocatalysis.

For most semiconductor photocatalysts, the improvements in photogenerated charge separation and charge utilization should typically be synchronized, to achieve high photocatalytic activity. Taking the model photocatalyst $\mathrm{BiVO}_{4}$ as an example, even though spatial charge separation between different facets has been achieved, the photocatalytic activity remains inadequate without the use of cocatalysts [13-15]. However, after depositing spatially separated dual cocatalysts onto various surfaces of $\mathrm{BiVO}_{4}$, the photocatalytic activity can be substantially enhanced by more than two orders of magnitude, highlighting the essential roles of dual cocatalysts and spatial charge separation in photocatalysis $[16,17]$. Similar phenomena have also been observed in various semiconductors, highlighting the advantages of rationally assembling appropriate cocatalysts in combination with their unique spatial charge separation properties [18-22]. These studies reveal that spatial charge separation strategies and appropriate cocatalysts are critical for simultaneously accelerating charge transfer and improving charge utilization in photocatalysis. Nonetheless, developing a more feasible approach to accelerate the interfacial charge separation and catalytic reactions on particulate photocatalysts remains a challenging but critical task. $\mathrm{Cd}_{0.9} \mathrm{Zn}_{0.1} \mathrm{~S}$ (CZS) is a typical visible-light-responsive photocatalyst, which has received considerable attention owing to its wide-range light absorption and suitable band structure for photocatalytic solar water splitting [23-25]. Nevertheless, the state-of-the-art efficiency of metal sulfides is very limited, owing to the imperfect separation and utilization of photogenerated charges [26-28]. Cocatalysts play an essential role in improving the performance of photocatalysts [29-31]. Although a number of precious metals, including $\mathrm{Pt}, \mathrm{Au}, \mathrm{Pd}$, and $\mathrm{AuPt}$, have been intensively tested to enhance surface redox reactions on metal-sulfide-based photocatalysts [32-35], the higher cost and limited supply of these high-quality metal-containing materials significantly slows down the practical implementation of photocatalyst technologies. Few studies have focused on integrating noble-metal-free cocatalysts on CZS-based photocatalysts and on developing interfacial charge separation strategies for simultaneously facilitating the charge separation and utilization processes.

Herein, we describe a feasible approach involving the surface assembly of earth-abundant cobalt oxide species on the outer surface of a CZS nanorod (NR) photocatalyst, to fabricate a hierarchical heterostructure. The resulting CZS@ $\mathrm{CoO}_{x}$ heterostructure exhibits effective interfacial electron-hole separation and accelerated surface reactions for photocatalytic hydrogen generation. These results highlight the advantages of integrating the charge separation strategy and appropriate cocatalysts for simultaneously improving the charge separation and surface reaction kinetics in photocatalysis.

\section{Experimental methods}

\subsection{Synthesis of CZS nanorods}

The CZS nanorods were produced via a reported facile hydrothermal approach [1]. Typically, $18.0 \mathrm{mmol}$ cadmium acetate dihydrate and $2.0 \mathrm{mmol}$ of zinc acetate dihydrate were added into a beaker, followed by $30 \mathrm{~mL}$, DI water, and $30 \mathrm{~mL}$ ethylenediamine stirred for half an hour to be uniformly soluble. Then, 25.0 mmol TAA was added and mixed for another half an hour. The subsequent mixture was poured into a Teflon-lined autoclave of $100 \mathrm{~mL}$ and heated at $220^{\circ} \mathrm{C}$ for $24 \mathrm{~h}$. The autoclave was naturally cooled after the reaction, and the resulting yellow material was cleaned with DI water and ethanol many times. Afterward, the cleaned yellow product was dried at $80^{\circ} \mathrm{C}$ for ten hours in a vacuum oven.

\subsection{Modification of CZS nanorods}

$100 \mathrm{mg}$ of CZS nanorods were spread out well around 40 $\mathrm{mL}$ of ethanol comprising $300 \mathrm{mg}$ of polyvinylpyrrolidone (PVP, MW $=40000$ ) and sonicated well to dispersed uniformly for $12 \mathrm{~h}$. The PVP modified CZS nanorods were washed with ethanol several times to collected PVP free CZS nanorods. After that, the obtained CZS nanorods were calcined at $100{ }^{\circ} \mathrm{C}$ for $8 \mathrm{~h}$.

\subsection{Synthesis of hierarchical CZS@CoOx core-shelled nanorods}

For the synthesis of hierarchical CZS@CoO ${ }_{x}$ core-shelled nanorods, $20 \mathrm{mg}$ of modified CZS nanorods were dispersed for half an hour in $20 \mathrm{~mL}$ ethanol and $60 \mathrm{~mL}$ DI water by sonication, accompanied by the addition of $0.4 \mathrm{mmol}$ $\mathrm{Co}\left(\mathrm{NO}_{3}\right)_{2} \cdot 6 \mathrm{H}_{2} \mathrm{O}$ as cobalt precursors. Then $0.7 \mathrm{mmol}$ hexamethylenetetramine was added, followed by $0.1 \mathrm{mmol}$ trisodium citrate added, and continued to stir for half an hour. The resulting mixture was then refluxed for various times $(3,5$, and $7 \mathrm{~h}$ ) with intense stirring in an oil bath at a $100{ }^{\circ} \mathrm{C}$. Then the product was collected through centrifugation after natural cooling to room temperature and rinsed many times using ethanol and dried for ten hours at $70{ }^{\circ} \mathrm{C}$ in a vacuum oven. To yield the hierarchical CZS@ $\mathrm{CoO}_{x}$ nanorods, the final product was heated at $300{ }^{\circ} \mathrm{C}$ for two hours with a heating rate of 5 ${ }^{\circ} \mathrm{C} / \mathrm{min}$. The $\mathrm{CoO}_{x}$ nanosheet was later synthesized by the same method, followed for the preparation of hierarchical CZS@ $\mathrm{CoO}_{x}$ Core-shelled without the addition of functionalized CZS nanorods.

\subsection{Synthesis of CZS-CoO $\mathrm{NRs}$ through solid state}

Briefly, a mixture of $20 \mathrm{mg}$ CZS and $0.4 \mathrm{~mm} \mathrm{Co}\left(\mathrm{NO}_{3}\right)_{2} \cdot 6 \mathrm{H}_{2} \mathrm{O}$ were grounded through agate mortar and put in an aluminum pot, heated under the same conditions as hierarchical nanorods in the synthesis of CZS@ $\mathrm{CoO}_{x}$. The bulk $\mathrm{CoO}_{x}$ material was thus produced by direct thermal annealing of cobalt nitrate hexahy- 
drate. Afterward, the resultant materials were grounded in a mortar into powder.

\subsection{Synthesis of CZS/CoO $\mathrm{NRs}$ through photodeposition}

The CZS nanorods powder $(0.1 \mathrm{~g})$, the essential quantity of $\mathrm{Co}\left(\mathrm{NO}_{3}\right)_{2} \cdot 6 \mathrm{H}_{2} \mathrm{O}$ solution and $\mathrm{NaIO}_{3}$ solution $(0.02 \mathrm{M}, 5 \mathrm{~mL})$ were mixed in $100 \mathrm{~mL}$ deionized water, and the suspension was then irradiated by a $300 \mathrm{~W}$ xenon lamp $(\lambda>420 \mathrm{~nm}, 150$ $\mathrm{mW} \mathrm{cm}-2$ ) under continuous stirring. After $3.5 \mathrm{~h}$ photo-deposition, the precipitate was isolated using centrifugation, washed with ethanol to more than 3 times, and finally dried at $60{ }^{\circ} \mathrm{C}$ overnight. Note here we have used an optimized amount of $\mathrm{CoO}_{x}(0.5 \%)$.

\subsection{Photocatalytic hydrogen evolution experiment}

The photocatalytic $\mathrm{H}_{2}$ evolution from water splitting was conducted in an online photocatalytic hydrogen production system (Perfect Light, Beijing CEL-SPH${ }_{2} \mathrm{~N}$ ). A photocatalytic reaction was performed by dissolving a powder sample of the catalyst (100 mg) in a $100 \mathrm{~mL}$ aqueous solution containing 0.10 $\mathrm{M} \mathrm{Na} 2 \mathrm{~S}-\mathrm{Na}_{2} \mathrm{SO}_{3}$ in the presence of visible light supplied from a lamp of $300 \mathrm{~W}$ Xe (Perfect Light, CEL-HXF-300) fitted with an optical source $(\lambda \geq 420 \mathrm{~nm})$ with a cutoff filter. The mixture was degassed first for $15 \mathrm{~min}$ by evacuation before the reaction to eliminate all the dissolved gases in reator. An online gas chromatograph (SP7800, TCD, molecular sieve $5 \AA$, $\mathrm{N}_{2}$ carrier) studied gaseous evolution photocatalytic reaction. the apparent quantum efficiency was determined under the same conditions, as followed during $\mathrm{H}_{2}$ production. In order to measure the light intensity to count the number of photons, we used a-Si photodiode (oreal $91105 \mathrm{~V}$ ). The Apparent quantum efficiency (AQE) was determined by using the equation below.

$$
\begin{aligned}
& \mathrm{AQY}[\%]=\frac{\text { Number of reacted electron }}{\text { Number } \text { incident photons }} \times 100 \\
& \mathrm{AQY}[\%]=\frac{\text { Number } \text { of evolved } H_{2} \times 2}{\text { Number } \text { incident photons }} \times 100
\end{aligned}
$$

\subsection{Photoelectrochemical measurements}

The fluorinated tin oxide (FTO) substratum was optimized to a scale of $(2 \mathrm{~cm}$ in length $0.5 \mathrm{~cm})$. The prepared substrate was then washed for 30 minutes with a cleaning agent, acetone solution, isopropyl alcohol, ethanol, and water under ultrasonic treatment. $3 \mathrm{mg}$ of the as synthesized photocatalyst with $40 \mu \mathrm{L}$ Nafion solution (5.0\% Nafion in ethanol) and $1000 \mu \mathrm{L}$ ethanol was dispersed by ultrasonication for $30 \mathrm{~min}$. Subsequently, the prepared ink was coated by spin coating on fluorinated tin oxide (FTO) glass electrode $(2 \mathrm{~cm} \times 0.5 \mathrm{~cm})$ and dried at $373 \mathrm{~K}$ for three hours in the air.

All photoelectrochemical tests were carried by an electrochemical workstation in a three-electrode device (Ivium Vertex One, Shanghai Chenhua, China). The irradiation source employed was a $300 \mathrm{~W}$ xenon lamp equipped with a filter $(\lambda \geq 420$ $\mathrm{nm}$ ), and $0.10 \mathrm{M}$ aqueous solution of $\mathrm{Na}_{2} \mathrm{SO}_{4}$ was used as an electrolyte. A Pt plate electrode is used as the counter electrode. A saturated calomel electrode (SCE) is used as the reference electrode, where the photocatalysts coated on FTO was used as working electrode.

\subsection{Characterization of materials}

The morphology of the prepared sample examined using a field-emission scanning electron microscope (FESEM; JEOL-6700) and a transmission electron microscope (TEM; JEOL, JEM-7700F). The crystal phases of the as prepared samples were evaluated by X-ray diffraction (XRD) on the Bruker D2 Phaser X-ray diffractometer with Ni-filtered Cu K5-007 radiation $(\lambda=1.5406 \AA)$ at a voltage of $30 \mathrm{kV}$ and a current of 10 $\mathrm{mA}$. Elemental mapping data were acquired using TEM (JEOL, JEM-7700F) to evaluate the materials composition. The samples chemical properties were determined using X-ray photoelectron spectra (XPS, PHI Quantum 2000) with a peak C1 relationship (284.6 eV). The sorption isotherms for $\mathrm{N}_{2}$ were obtained from the ASAP2020 M device. Samples were equilibrated in a vacuum at $120{ }^{\circ} \mathrm{C}$ for four hours and instead evaluated at $77 \mathrm{~K}$ to determine $\mathrm{N}_{2}$ sorption. UV-vis diffuse reflectance performed using Varian Cary 500 UV-Vis spectrometer, where $\mathrm{BaSO}_{4}$ was used as a reference. Photoluminescence (PL) spectra were recorded using Edinburgh Analytical Instruments FL/FSTCSPC920, including a time-correlated single-photo-counting system at room temperature.

\section{Results and discussion}

The CZS@CoO ${ }_{x}$ hierarchical heterostructures were synthesized using CZS nanorods as precursor. The surface of the hydrothermally prepared CZS NRs was modified using PVP to modulate the interfacial energy, enabling the complete encapsulation of the nanorods by an oxide layer and preventing their aggregation; this was followed by the fabrication of cobalt species $\left(\mathrm{CoO}_{x}\right)$ on the external surface of the CZS nanorods through a solvothermal process, as shown in Fig. S1. The CZS nanorods show well-aligned and sharp X-ray diffraction peaks indexed to the wurtzite structure (Fig. S2). The field-emission scanning electron microscopy (FESEM) and transmission electron microscopy (TEM) images in Fig. 1 show that the cobalt species effectively encapsulate the CZS nanorods, achieving a hierarchical core-shell arrangement. The $\mathrm{CoO}_{x}$ species are evenly coated on the entire surface of the CZS nanorods, establishing an ordered CZS@CoO ${ }_{x}$ core-shell hierarchical heterostructure (Fig. S3). The closer observation of the $\mathrm{CoO}_{x}$ shell surrounding the CZS nanorods shows that no visible intershell gap is present (Fig. S4), indicating a compact contact between the CZS core and the $\mathrm{CoO}_{x}$ shell. The high-resolution transmission electron microscopy (HRTEM) image shows the crystalline structure of the as-synthesized sample, while the fringes with a lattice parameter of $0.338 \mathrm{~nm}$ match well with the (002) hexagonal plane of CZS (Fig. 1(f)). The lattice distance of $\sim 0.283 \mathrm{~nm}$ on the outermost surface can be assigned to the (311) cubic plane of $\mathrm{Co}_{3} \mathrm{O}_{4}$, while the crystal lattice spacing of $0.236 \mathrm{~nm}$ correlates well with the (200) cubic plane of CoO [36]. Moreover, the ele- 


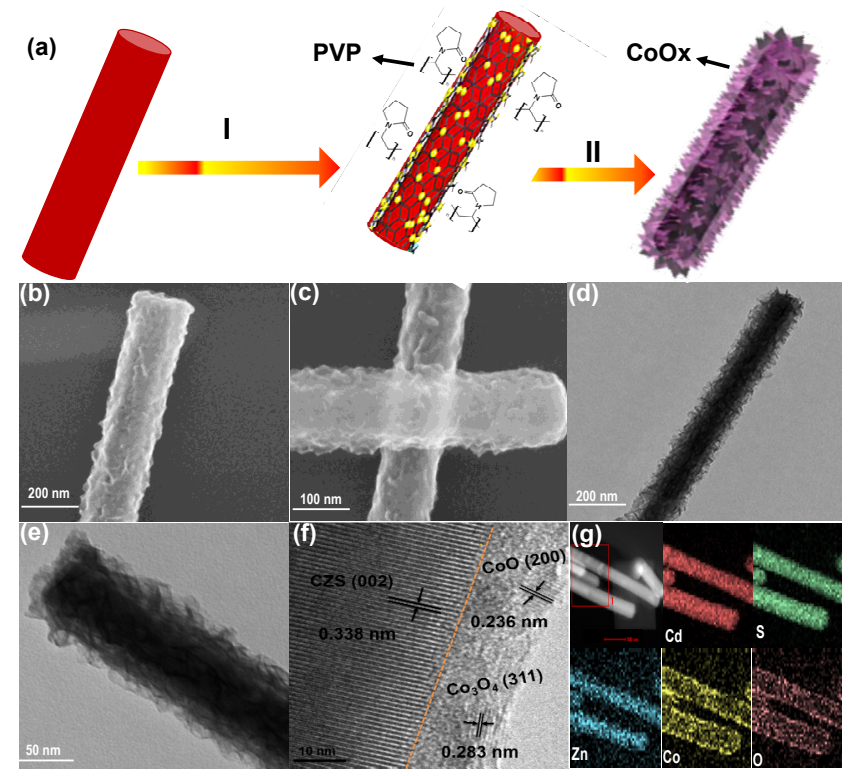

Fig. 1. (a) Schematic illustration of the functionalization of CZS nanorods for synthesizing hierarchical CZS@ $\mathrm{CoO}_{x}$ heterostructured nanorods; (b,c) FESEM images of CZS and CZS@ $@ \mathrm{CoO}_{x} ;(\mathrm{d}, \mathrm{e})$ TEM images of hierarchical CZS@CoO ${ }_{x}$ nanorods; (f) HRTEM image of CZS@CoO $;$; (g) elemental mappings of individual CZS@ $\mathrm{CoO}_{x}$ nanorods.

mental mappings of the CZS@ $\mathrm{CoO}_{x}$ heterostructured nanorods show that the $\mathrm{CoO}_{x}$ shell is uniformly coated on the entire nanostructure (Fig. 1(g)). The coherent epitaxial growth of $\mathrm{CoO}_{x}$ on the CZS nanorods suggests the development of a special interfacial contact, which is highly beneficial for effective charge transport in the composite photocatalyst.

In addition, the $\mathrm{N}_{2}$ sorption isotherms and the related pore size distribution curves reveal the presence of mesopores in CZS@CoO ${ }_{x}$ (Fig. S5), which can stimulate the mass transfer in photocatalysis. In hierarchical photocatalysts, the porous network is effectively interconnected with various pore sizes and dimensions, such that the reactant species can rapidly transfer to the active sites and the products can also move freely. In addition, the mass ratio of CZS to $\mathrm{CoO}_{x}$ in the $\mathrm{CZS} @ \mathrm{CoO}_{x}$ heterostructures can also be adjusted by modifying the amount of CZS nanorods in the reaction vessel. The thickness of the $\mathrm{CoO}_{x}$ species can be controlled by varying the concentrations of precursors and the reaction conditions, without altering their uniform distribution (Fig. S6).

Further analyses of the surface chemical states of each element present in the composites were carried out to understand the interactions between $\mathrm{CoO}_{x}$ and CZS, as illustrated by the high-resolution X-ray photoelectron spectroscopy (XPS) profiles (Fig. 2 and S7). The binding energies corresponding to the $\mathrm{Cd} 3 d_{3 / 2}$ and $\mathrm{Cd} 3 d_{5 / 2}$ states of divalent cadmium $\left(\mathrm{Cd}^{2+}\right)$ appear at 411.78 and $405.08 \mathrm{eV}$ [37]. The $\mathrm{Zn} 2 p$ peaks at 1021.85 and $1045.04 \mathrm{eV}$ can be attributed to $\mathrm{Zn}^{2+}$, while the signals at 161.50 and $162.67 \mathrm{eV}$ can be assigned to $\mathrm{S}^{2-}$ species [38]. Interestingly, the binding energies of the $\mathrm{Cd} 3 d, \mathrm{Zn} 2 p$, and S $2 p$ electrons shift toward the left by $0.75,0.70$, and $0.75 \mathrm{eV}$, respectively, compared to those of bare CZS. These results highlight the strong interaction between the CZS core and the $\mathrm{CoO}_{x}$ shell. The similar binding energies of the oxidation states of cobalt, multiplet splitting, and the presence of satellites should be considered when examining the XPS profiles of the Co $2 p$ core levels. It is easy to assign the surface chemical states of cobalt by considering satellite peaks instead of basic core levels [39]. The satellite peak near the $\mathrm{Co}_{3} \mathrm{O}_{4} 2 p_{3 / 2}$ signal is higher than that of $\mathrm{CoO}$, while the satellite peak corresponding to the $\mathrm{Co}_{3} \mathrm{O}_{4} 2 p_{1 / 2}$ level is slightly lower than the $\mathrm{CoO}$ signal [40]. As can be seen in Fig. $2(\mathrm{~d})$, the Co $2 p_{3 / 2}$ and $2 p_{1 / 2}$ binding energies of 780.12 and $795.6 \mathrm{eV}$ are different from those of the single-phase $\mathrm{CoO}$ or $\mathrm{Co}_{3} \mathrm{O}_{4}$; however, the two main peaks can be
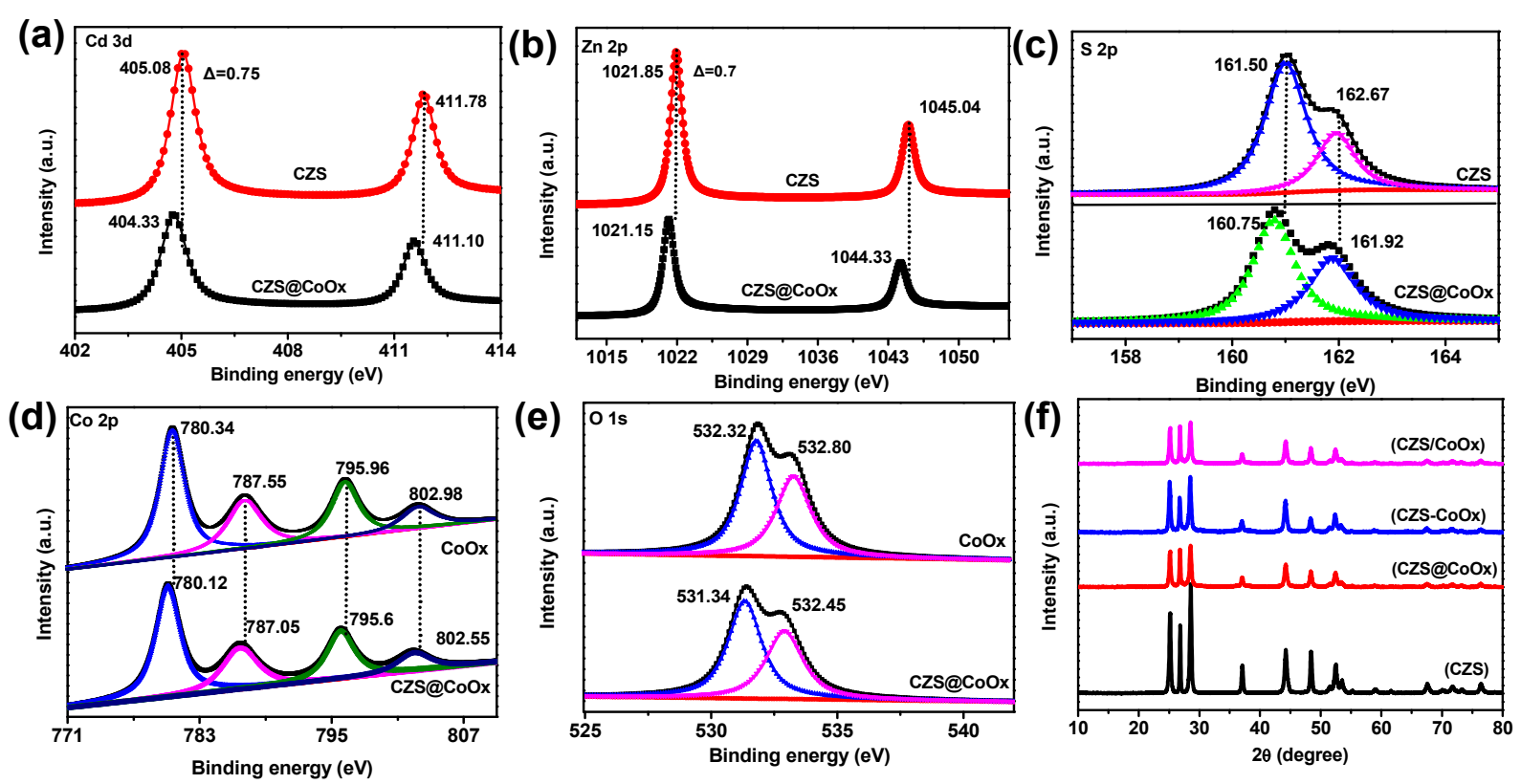

Fig. 2. Structural and compositional characterizations of the photocatalysts. (a-e) High resolution XPS spectra of CZS@CoO $2 p$, (d) Co 2p, (e) $01 s$; (f) XRD patterns of CZS and CZS@CoO ${ }_{x}$ hierarchical nanorods. 
assigned to $\mathrm{Co}_{3} \mathrm{O}_{4}$ [41]. In comparison, the two satellite peaks can be considered the characteristics of $\mathrm{CoO}$ [42]. Comparison

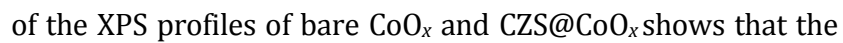
CZS@ $\mathrm{CoO}_{x} 2 p_{1 / 2}$ and $2 p_{3 / 2}$ peaks shift by a certain degree toward negative binding energies, denoting a strong connectivity between the $\mathrm{CoO}_{x}$ shell and the CZS core. The interfacial bonds formed between Co and $\mathrm{S}$ atoms promote the efficient extraction of light-induced electrons from $\mathrm{CoO}_{x}$ to $\mathrm{CZS}$ [43]. The phase composition, purity, and crystallinity of the hexagonal CZS nanorods remain unchanged after introducing $\mathrm{CoO}_{x}$ species on the surface (Fig. 2(f)).

Diffuse reflectance spectroscopy (DRS) was performed to evaluate the impact of $\mathrm{CoO}_{x}$ on the optical behavior of CZS (Fig. S8(a)). The main CZS absorption is observed at $\sim 516 \mathrm{~nm}$, while those of the $\mathrm{CZS}-\mathrm{CoO}_{x}$ and $\mathrm{CZS} / \mathrm{CoO}_{x}$ hybrid nanocomposites show a slight red shift up to $\sim 522$ and $\sim 524 \mathrm{~nm}$, and that of CZS@ $\mathrm{CoO}_{x}$ extends to $\sim 538 \mathrm{~nm}$; the corresponding bandgaps are $2.48,2.45,2.44$, and $2.38 \mathrm{eV}$, respectively, as shown in the Tauc plot (Fig. S8(b)). The CZS@ $\mathrm{CoO}_{x}$ hierarchical nanoarchitecture exhibits an enhanced visible light absorption, attributed to the characteristic absorption of $\mathrm{CoO}_{x}$ coated on the CZS nanorods. As the amount of deposited $\mathrm{CoO}_{x}$ increases, the absorption edge shows a red shift in the visible region. This may be because $\mathrm{CoO}_{x}$ captures the photogenerated holes and electrons are left in the CZS NRs, creating an electron-rich state and reducing the electron transition energy in the CZS@CoO ${ }_{x}$ photocatalyst. Mott-Schottky plots of the materials were obtained to determine their conduction band (CB) positions (Fig. S9). Moreover, the flat-band potentials of CZS and $\mathrm{CoO}_{x}$ were -0.62 and $1.92 \mathrm{~V} v s$. SCE, respectively. Hierarchical nanocomposites have been extensively studied in various areas of semiconduc- tor photocatalysis owing to their unique features, such as enhanced light harvesting, effective separation of charges, mass transfer, and adsorption capabilities.

In order to study the effect of the $\mathrm{CoO}_{x}$ species on the separation of photogenerated electron-hole pairs, we carried out steady-state photoluminescence (PL) spectroscopy measurements. As illustrated in Fig. 3(a) and 3(b), the CZS and CZS@ $\mathrm{CoO}_{x}$ composites exhibit broad emission peaks at approximately $540 \mathrm{~nm}$, corresponding to the band-to-band transitions of the samples [44]. For comparison, two different composites prepared by solid-state reaction and photodeposition, denoted as $\mathrm{CZS}-\mathrm{CoO}_{x}$ and $\mathrm{CZS} / \mathrm{CoO}_{x}$, respectively, were also examined (Fig. S10). The emission of CZS@ $\mathrm{CoO}_{x}$ is slightly weaker than those of $\mathrm{CZS}$, CZS- $\mathrm{CoO}_{x}$, and $\mathrm{CZS} / \mathrm{CoO}_{x}$, indicating that the recombination of light-induced charge carriers in the CZS@ $\mathrm{CoO}_{x}$ hierarchical nanorods is effectively suppressed by creating a specific interface between CZS and $\mathrm{CoO}_{x}$. This is due to the rapid transmission of photoinduced charges, resulting in the creation of efficient networks for the transport of these charges to the active spots on the porous walls of the composite photocatalyst.

Although the PL intensities of CZS- $\mathrm{CoO}_{x}$ and $\mathrm{CZS} / \mathrm{CoO}_{x}$ are lower than those of pristine CZS, they are substantially higher than those of CZS@ $\mathrm{CoO}_{x}$, which is expected to be closely correlated with the interfacial contact between the CZS and $\mathrm{CoO}_{x}$ species. The quenched PL intensity may be attributed to the hole-capturing effect of $\mathrm{CoO}_{x}$, resulting in more efficient electron-hole pair separation. To gain some insight into the effi-

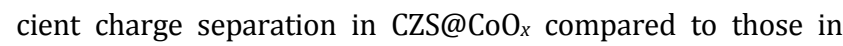
$\mathrm{CZS} / \mathrm{CoO}_{x}$ and $\mathrm{CZS}-\mathrm{CoO}_{x}$, various photo/electrochemical characterizations were carried out. Time-resolved photolumines- (a)

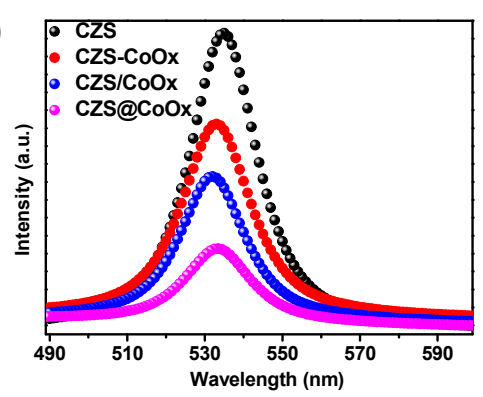

(b)

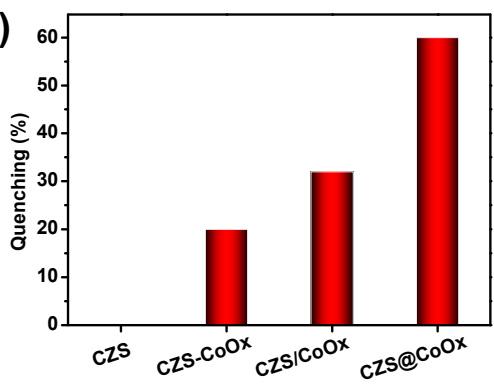

(d)
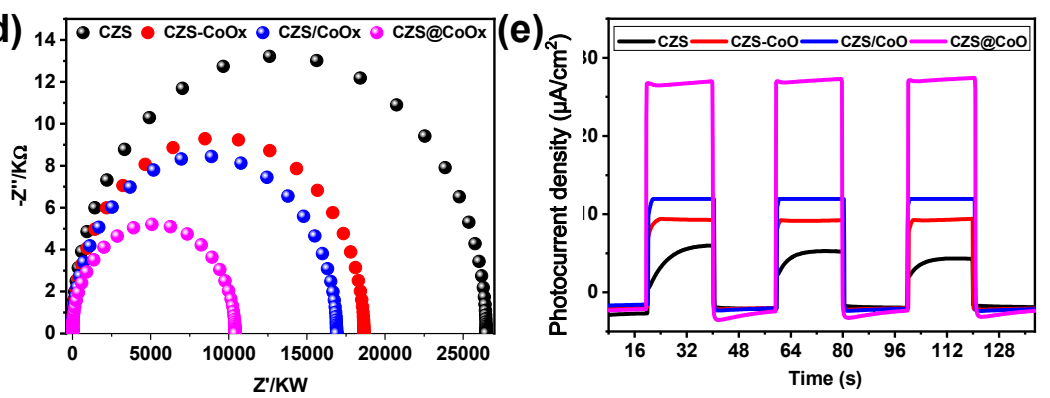
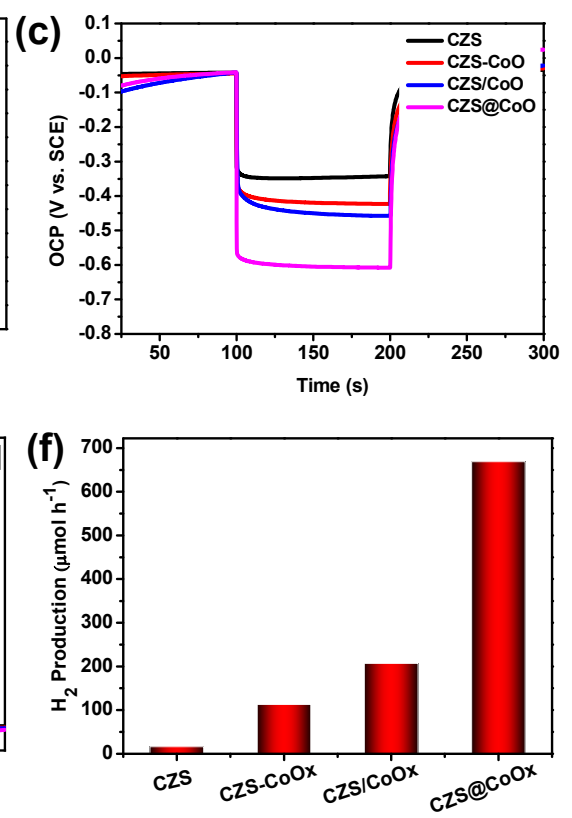

Fig. 3. (a) Steady-state PL spectra of the CZS samples; (b) PL emission spectra and percentage quenching of $\mathrm{CZS} \mathrm{CZS}-\mathrm{CoO} \mathrm{O}_{x}, \mathrm{CZS} / \mathrm{CoO}_{x}$, and $\mathrm{CZS} @ \mathrm{CoO}{ }_{x}$;

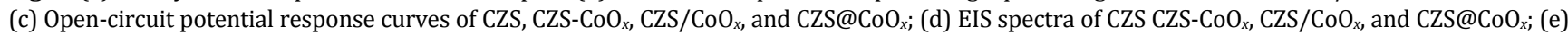
Transient photocurrent of CZS CZS-CoO, $\mathrm{CZS} / \mathrm{CoO}_{x}$, and CZS@CoO ; (f) Comparative photocatalytic $\mathrm{H}_{2}$ production activities of different CZS samples. Condition: catalyst, $100 \mathrm{mg} ; 0.10 \mathrm{M} \mathrm{Na} 2 \mathrm{~S}-\mathrm{Na}_{2} \mathrm{SO}_{3}$ aqueous solution (100 mL); light source: Xe lamp (300 W) with an optical filter ( $\left.\lambda \geq 420 \mathrm{~nm}\right)$. 
cence (TRPL) spectroscopy was employed to probe the charge carrier dynamics of the materials, as shown in Fig. s11 and Table S1. The decay kinetics of CZS@ $\mathrm{CoO}_{x}$ exhibits a longer average lifetime $(6.17 \mathrm{~ns})$ than those of $\mathrm{CZS}-\mathrm{CoO}_{x}$ and $\mathrm{CZS} / \mathrm{CoO}_{x}$ (4.17 and $4.62 \mathrm{~ns}$, respectively), which indicates that $\mathrm{CZS@ \textrm {CoO } _ { x }}$ can effectively facilitate the isolation of photogenerated electron-hole pairs. In light of the above results, various photoelectrochemical characterizations, including open-circuit potential (OCP) measurements, were performed to further analyze the impact of $\mathrm{CoO}_{x}$ on the separation of photogenerated charges. The OCP measurements were carried out by recording the open-circuit photovoltage under illumination, followed by switching off the light, as shown in Fig. 3(c). The OCP reflects differences between photoanodes and counter electrodes at the Fermi level. Redox equilibration determines the electrode potential in the absence of light irradiation, as the holes are scavenged at the interface and the electrons accumulate within the photoanodes composed of CZS, CZS-CoO, $\mathrm{CZS} / \mathrm{CoO}_{x}$, and CZS@ $\mathrm{CoO}_{x}$. Electron accumulation allows the Fermi level to change to even more negative potentials, leading to an improved OCP. The open-circuit voltage is as high as the concentration of electrons that can compete with the recombination of charges and achieve a steady state. When the accumulation of electrons reaches the steady state, the increase in photovoltage slows down, due to the competition with the charge recombination $[45,46]$. Therefore, the improved OCP reaction demonstrates that the incorporation of $\mathrm{CoO}_{x}$ into the CZS nanorods in the form of a hierarchical architecture enhances the separation of photoinduced electrons and holes, compared to $\mathrm{CZS}-\mathrm{CoO}_{x}$ and $\mathrm{CZS} / \mathrm{CoO}_{x}$ samples fabricated by impregnation and photodeposition. The enhanced open-circuit voltage response is attributed to the uniform and compact interfacial contact of the hierarchical CZS@ $\mathrm{CoO}_{x}$ heterostructure. Based on the obtained results, this efficient performance is attributed to the hierarchical architecture, which has the following unique characteristics: (1) improved charge carrier distribution efficiency, achieved by reducing the overall thickness of the semiconductor; (2) enhanced charge transmission, obtained by creating a large surface area for the interaction with electrolytes, which results in band bending within the semiconductor; (3) enabled formation of heterojunctions through the addition of a second semiconductor material.

Furthermore, the electrochemical impedance spectroscopy (EIS) curves show that $\mathrm{CZS@} \mathrm{CoO}_{x}$ displays a much smaller high-frequency semicircle, denoting lower charge-transfer resistance than those of CZS-CoO $x$ and $\mathrm{CZS} / \mathrm{CoO}_{x}$, which ensures faster transfer of photogenerated charges at the interface (Fig. 3(d)). The uniform distribution of $\mathrm{CoO}_{x}$ leads to significantly improved charge separation and transfer of photogenerated carriers, which is in good agreement with the OCP results. In addition, the transient photocurrent spectra display a marked

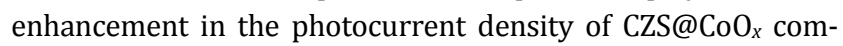
pared to those of CZS, CZS- $\mathrm{CoO}_{x}$, and $\mathrm{CZS} / \mathrm{CoO}_{x}$, implying improved separation and transfer of photogenerated charge carriers in the CZS@ $\mathrm{CoO}_{x}$ hierarchical nanorod photocatalyst (Fig. $3(\mathrm{e})$ ). Based on the above results, it can be concluded that the hierarchical CZS@ $\mathrm{CoO}_{x}$ heterostructure plays a significant role in the interfacial separation and transport of photogenerated charges. The performance of the photocatalysts was further monitored by measuring the generation of hydrogen from an aqueous solution containing $\mathrm{Na}_{2} \mathrm{~S}$ and $\mathrm{Na}_{2} \mathrm{SO}_{3}$ as the sacrificial electron donors under visible-light irradiation $(\lambda \geq 420 \mathrm{~nm})$. The pristine CZS exhibits a relatively low $\mathrm{H}_{2}$ evolution activity. However, after depositing a thin layer of $\mathrm{CoO}_{x}$ onto the CZS sample to form a hierarchical heterostructure, the photocatalytic $\mathrm{H}_{2}$ production is substantially enhanced (more than 100-fold increase) with respect to the CZS sample (Fig. 3(f) and S12). This noble-metal-free photocatalyst exhibits an extremely high rate of hydrogen generation, even higher than those of noble metal systems (Table S2). In comparison, the photocatalytic $\mathrm{H}_{2}$ production of the $\mathrm{CZS}-\mathrm{CoO}_{x}$ and $\mathrm{CZS} / \mathrm{CoO}_{x}$ nanocompo-

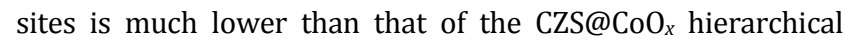
nanorods, demonstrating the advantages of the CZS@CoO ${ }_{x}$ hierarchical heterostructure in photocatalysis (Fig. 3(f)). The stability of the synthesized CZS@CoO ${ }_{x}$ hierarchical nanostructure was evaluated by monitoring its $\mathrm{H}_{2}$ production for $12 \mathrm{~h}$. Fig. S13 shows that the $\mathrm{CZS} @ \mathrm{CoO}_{x}$ photocatalyst maintains remarkable stability over four consecutive cycles, without any sign of deactivation. The uniform and compact interfacial contact is expected to play a significant role in enabling the separation of photogenerated charges, resulting in an enhanced photocatalytic $\mathrm{H}_{2}$ generation. To understand the role of the hierarchical morphology, the photocatalytic $\mathrm{H}_{2}$ production of bare $\mathrm{CoO}_{x}$ and $\mathrm{Pt} / \mathrm{CoO}_{x}$ prepared without CZS was compared with that of CZS@CoO $x$, as shown in Fig. S14. The analysis shows that the bare $\mathrm{CoO}_{x}$ and Pt-containing $\mathrm{CoO}_{x}$ display negligible $\mathrm{H}_{2}$ production compared to the hierarchical core-shell CZS@CoO The hierarchical composite photocatalyst not only exposes more active sites, but also facilitates electron transport, which results in excellent photocatalytic activity $[47,48]$.

Pt is commonly employed as an efficient cocatalyst for photocatalytic $\mathrm{H}_{2}$ production because of its large work function [49-51]; therefore, the $\mathrm{H}_{2}$ evolution performances of the Pt/CZS and Pt/CZS@CoO ${ }_{x}$ composites were also investigated under identical conditions. Although the photocatalytic $\mathrm{H}_{2}$ production activity is considerably enhanced after depositing $\mathrm{Pt}$ as a cocatalyst on the CZS, the Pt/CZS sample still displays a much lower activity than CZS@ $\mathrm{CoO}_{x}$. The deposition of Pt nanoparticles on the CZS@ $\mathrm{CoO}_{x}$ hierarchical photocatalyst results in a two-fold increase in the photocatalytic $\mathrm{H}_{2}$ production. This increased $\mathrm{H}_{2}$ production is attributed to the compact interfacial contact, which facilitates the directional charge flow to the $\mathrm{Pt}$ nanoparticles, effectively trapping the photoinduced electrons for water reduction, as shown in Fig. 4(a). The apparent quantum efficiency (AQE) of the hierarchical CZS@CoO $x$ was estimated to be higher than $\sim 20 \%$ for photocatalytic $\mathrm{H}_{2}$ production under $420-\mathrm{nm}$ visible light irradiation. This is one of the highest AQE values measured for visible-light-driven $\mathrm{H}_{2}$ generation on noble-metal-free photocatalysts. Further introduction of the Pt cocatalyst results in the AQE increasing from 20\% to $37 \%$ at $420 \mathrm{~nm}$, under the same conditions. Steady-state PL spectra and OCP measurements were also performed to identify the origin of the improved photocatalytic performances. As shown in Fig. 4(b) and 4(c), the bandgap PL emissions exhibit 
(a)

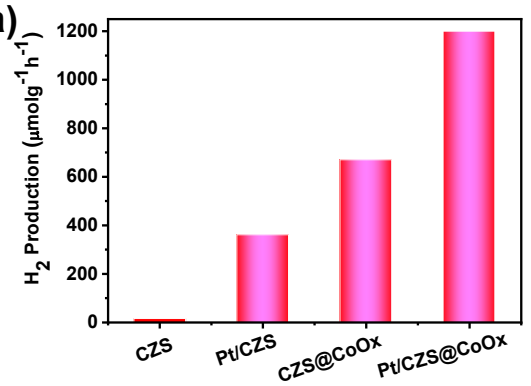

(d)

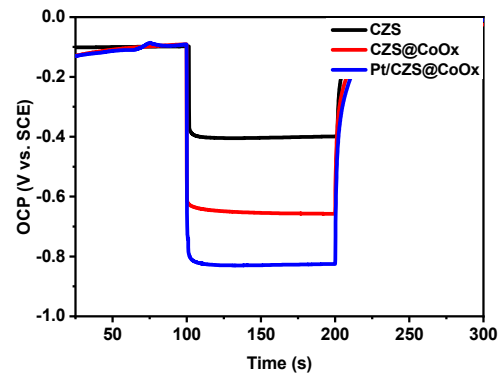

(b)

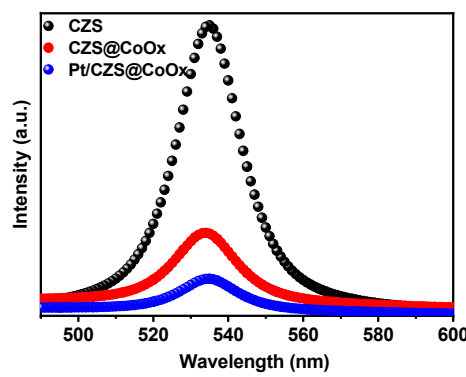

(e)

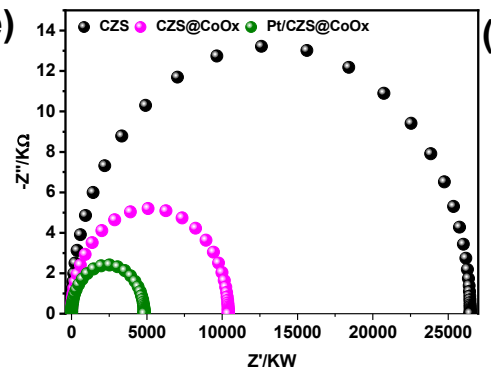

(c)

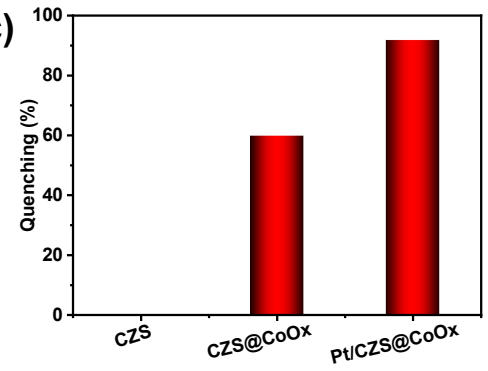

(f)

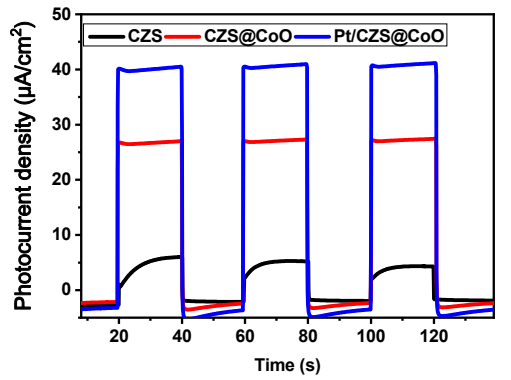

Fig. 4. (a) Photocatalytic $\mathrm{H}_{2}$ production activities of CZS based photocatalysts; Condition: catalyst $100 \mathrm{mg} ; 0.10 \mathrm{M} \mathrm{Na}_{2} \mathrm{~S}_{-} \mathrm{Na}_{2} \mathrm{SO}_{3}$ aqueous solution (100 $\mathrm{mL}$ ); Light source: Xe lamp (300W) with an optical filter $(\lambda \geq 420 \mathrm{~nm}$ ). (b) Steady-state PL spectra of the samples; (c) PL emission spectra and percentage quenching of CZS, CZS@CoO , and Pt/CZS@CoO ${ }_{x}$ (d) Open-circuit potential response curves of CZS, CZS@CoO $x$, and Pt/CZS@CoO spectra of CZS, CZS@CoO $\mathrm{C}_{x}$ and Pt/CZS@CoO ${ }_{x}$ (e) Transient photocurrent of CZS, $\mathrm{CZS@CoO}$ and Pt/CZS@CoO

an obvious decline, and the PL intensity can be significantly quenched by incorporating $\mathrm{CoO}_{x}$ onto the CZS nanorods, reflecting a decrease in the recombination of photogenerated carriers. Moreover, the PL intensity is further weakened after the introduction of Pt nanoparticles and shows a negligible PL intensity, which is further reflected by the OCP measurements (Fig. 4(d)). The Pt/CZS@CoO $x$ sample displays the smallest semicircle in the Nyquist plots obtained from the EIS measurements (Fig. 4(e)), reflecting its lowest charge transport resistance, allowing fast separation and transport of photoinduced charges. The improved photogenerated separation of charge carriers in Pt/CZS@CoO ${ }_{x}$ is further demonstrated by a substantial improvement in its transient photocurrent spectrum, reflecting an enhanced separation of photogenerated charges in the Pt/CZS@CoO ${ }_{x}$ nanostructures in comparison to

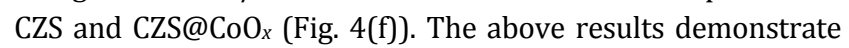
that the CZS@CoO $x$ hierarchical nanorods can enhance the separation and transfer of photogenerated charge carriers and optimize the kinetics of catalytic reactions, resulting in highly efficient photocatalytic systems.
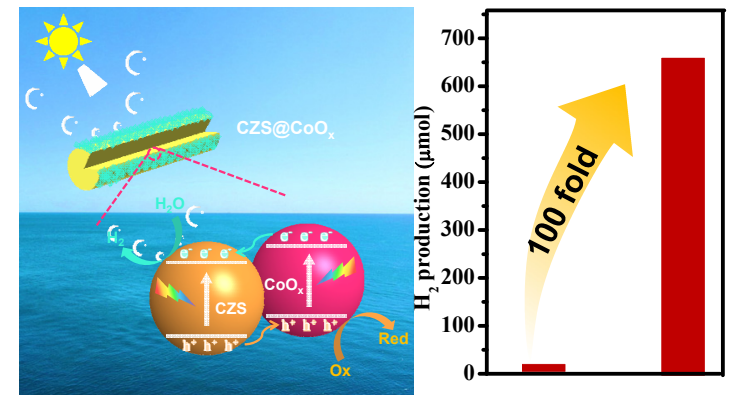

Fig. 5. Schematic illustration of the proposed mechanism in the hierarchical heterojunction architectures.
A graphical representation of the proposed photocatalytic

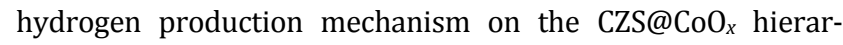
chical core-shell structure is shown in Fig. 5. The $\mathrm{CoO}_{x}$ species are uniformly coated on the CZS nanorods. The difference between the Fermi levels of $\mathrm{CoO}_{x}$ and CZS determines a viable thermodynamic charge separation path when $\mathrm{CoO}_{x}$ is in contact with CZS, which provides the driving force for promoting the interfacial charge transfer. Upon light irradiation, the directional flow of photoinduced electrons from $\mathrm{CoO}_{x}$ to the CZS starts, while the holes generated after light irradiation migrate from CZS to $\mathrm{CoO}_{x}$. As a result, a staggered gap with type II alignment is created by the band structure match between CZS and $\mathrm{CoO}_{x}$ [52]. Furthermore, the $\mathrm{CoO}_{x}$ species may also act as efficient cocatalysts for trapping the photogenerated charges and creating reactive sites promoting the charge utilization process. Hence, the unique $\mathrm{CZS} @ \mathrm{CoO}_{x}$ hierarchical structure simultaneously integrates efficient charge separation and charge utilization processes through the surface assembly of $\mathrm{CoO}_{x}$ species, which significantly enhances the performance of the CZS photocatalyst.

\section{Conclusions}

In conclusion, herein we report a facile surface assembly approach to fabricate hierarchical CZS@CoO ${ }_{x}$ heterostructured nanorods. The CZS@CoO ${ }_{x}$ heterostructure with an ultrathin layer of $\mathrm{CoO}_{x}$ can effectively enhance the kinetics of surface reactions and simultaneously reduce the bulk charge recombination of the CZS photocatalyst. The epitaxial growth of ultrathin $\mathrm{CoO}_{x}$ species, creating bonded interface states on the hierarchical CZS@CoO $x$ nanorods, can efficiently facilitate the interfacial charge separation. At the same time, the surface 
cobalt species also act as a cocatalyst for accelerating the charge-based photocatalytic reactions, significantly enhancing the photocatalytic $\mathrm{H}_{2}$ generation under visible light. Our findings demonstrate the advantages of rationally designing the architecture and interface structure of photocatalysts to simultaneously facilitate the charge separation and surface catalytic reactions in photosynthetic solar energy conversion.

\section{Acknowledgments}

The authors would like to thank Abraham Abdul Adenle and Bin Zeng for useful discussions.

\section{Electronic supporting information}

Supporting information is available in the online version of this article.

\section{References}

[1] N. Armaroli, V. Balzani, Angew. Chem. Int. Ed., 2007, 46, 52-66.

[2] Z. Wang, C. Li, K. Domen, Chem. Soc. Rev., 2019, 48, 2109-2125.

[3] M. S. Faber, S. Jin, Energy Environ. Sci., 2014, 7, 3519-3542.

[4] J. Gong, C. Li, M. R. Wasielewski, Chem. Soc. Rev., 2019, 48, 1862-1864.

[5] N. S. Lewis, Science, 2016, 351, aad1920.

[6] C. Zhou, S. Wang, Z. Zhao, Z. Shi, S. Yan, Z. Zou, Adv. Funct. Mater., 2018, 28, 1801214.

[7] L. Xiao, L. Zhuang, Y. Liu, J. Lu, H. D. Abruna, J. Am. Chem. Soc., 2009, 131, 602-608.

[8] Y. Gao, J. Zhu, H. An, P. Yan, B. Huang, R. Chen, F. Fan, C. Li, J. Phys. Chem. Lett., 2017, 8, 1419-1423.

[9] S. Wang, G. Liu, L. Wang, Chem. Rev., 2019, 119, 5192-5247.

[10] G. Yang, B. Yang, T. Xiao, Z. Yan, Appl. Surf. Sci., 2013, 283, 402-410

[11] M. Luo, Y. Liu, J. Hu, H. Liu, J. Li, ACS Appl. Mater. Interf., 2012, 4, 1813-1821.

[12] G. Ai, H. Li, S. Liu, R. Mo, J. Zhong, Adv. Funct. Mater., 2015, 25,
5706-5713.

[13] J. Zhu, F. Fan, Chen, R. H. An, Z. Feng, C. Li, Angew. Chem. Int. Ed., 2015, 127, 9239-9242.

[14] T. Tachikawa, T. Ochi, Y. Kobori, ACS Catal., 2016, 6, 2250-2256.

[15] J. Zhu, S. Pang, T. Dittrich, Y. Gao, W. Nie, J. Cui, R. Chen, H. An, F. Fan, C. Li, Nano Lett., 2017, 17, 6735-6741.

[16] R. Li, H. Han, F. Zhang, D. Wang, C. Li, Energy Environ Sci., 2014, 7, 1369-1376.

[17] R. Li, F. Zhang, D. Wang, J. Yang, M. Li, J. Zhu, X. Zhou, H. Han, C. Li, Nat. Commun., 2013. 4, 1432.

[18] R. Chen, S. Pang, H. An, J. Zhu, S. Ye, Y. Gao, F. Fan, C. Li, Nat. Energy, 2018, 3, 655-663.

[19] R. Li, X. Tao, R. Chen, F. Fan, C. Li, Chem. Eur. J., 2015. 21, 14337-14341.

[20] L. Mu, Y. Zhao, A. Li, S. Wang, Z. Wang, J. Yang, Y. Wang, T. Liu, R. Chen, J. Zhu, F. Fan, R. Li, C. Li, Energy Environ. Sc., 2016, 9, 2463-2469.

[21] Q. Zhang, R. Li, Z. Li, A. Li, S. Wang, Z. Liang, S. Liao, C. Li, J. Catal., 2016, 337, 36-44.

[22] Q. Zhang, Z. Li, S. Wang, R. Li, X. Zhang, Z. Liang, H. Han, S. Liao, C. Li, ACS Catal., 2016, 6, 2182-2191.

[23] J. Ran, B. Zhu, S. Z. Qiao, Angew. Chem. Int. Ed., 2017, 56, 10373-10377

[24] H. F. Ye, R. Shi, X. Yang, W. F. Fu, Y. Chen, Appl. Catal. B, 2018, 233, 70-79.

[25] B. J. Ng, L. K. Putri, X. Y. Kong, P. Pasbakhsh, S. P. Chai, Appl. Catal. $B, 2020,262,118309$.

[26] M. Liu, Y. Chen, J. Su, J. Shi, X. Wang, L. Guo, Nat. Energy, 2016, 1, 16151.

[27] G. Yu, J. Qian, P. Zhang, B. Zhang, W. Zhang, W. Yan, G. Liu, Nat. commun., 2019, 10, 4912.

[28] G. Yu, W. Zhang, Y. Sun, T. Xie, A. M., Ren, X. Zhou, G. Liu. J. Mater. Chem. A, 2016, 4, 13803-13808.

[29] J. Yang, D. Wang, H. Han, Can, Li, Acc. Chem. Res., 2013, 46, 1900-1909.

[30] P. Zhang, T. Wang, X. Chang, J. Gong, Acc. Chem. Res., 2016, 49, 911-921.

[31] N. Xiao, S. Li, X. Li, L. Ge, Y. Gao, N. Li, Chin. J. Catal, 2020, 41, 642-671.

[32] L. Amirav, A. P. Alivisatos, J. Phys. Chem. Lett., 2010, 1,

\section{Graphical Abstract}

Chin. J. Catal., 2021, 42: 1004-1012 doi: 10.1016/S1872-2067(20)63717-2

\section{Surface assembly of cobalt species for simultaneous} acceleration of interfacial charge separation and catalytic reactions on $\mathrm{Cd}_{0.9} \mathrm{Zn}_{0.1} \mathrm{~S}$ photocatalyst

Khakemin Khan, Lifen Xu, Ming Shi, Jiangshan Qu, Xiaoping Tao, Zhaochi Feng, Can Li, Rengui Li*

Dalian Institute of Chemical Physics, Chinese Academy of Sciences, Dalian National Laboratory for Clean Energy;

University of Chinese Academy of Sciences

The hierarchical CZS@CoO heterostructure, synthesized via surface assembly of $\mathrm{CoO}_{x}$ species on the surface of CZS, exhibits efficient interfacial charge separation and accelerated surface HER activity.

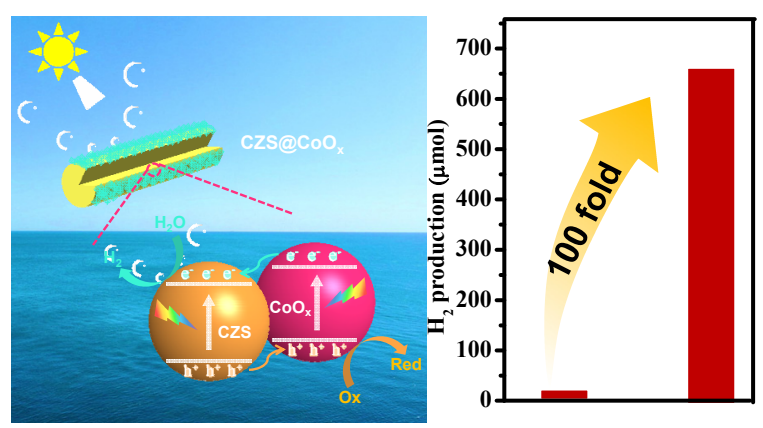


1051-1054.

[33] X. Ma, K. Zhao, H. Tang, Y. Chen, C. Lu, W. Liu, Y. Gao, H. Zhao, Z. Tang, Small, 2014, 10, 4664-4670.

[34] H. Zhou, J. Pan, L. Ding, Y. Tang, J. Ding, Q. Guo, T. Fan, D. Zhang, Int. J. Hydrogen Energy, 2014, 39, 16293-16301.

[35] J. Xu, T. Zhao, Z. Liang, L. Zhu, Chem. Mater., 2008, 20, 1688-1690.

[36] S. Chen, S. Shen, G. Liu, Y. Qi, F. Zhang, C. Li, Angew. Chem. Int. Ed., 2015, 127, 3090-3094.

[37] Y. Liu, S. Ding, Y. Shi, X. Liu, Z. Wu, Q. Jiang, T. Zhou, N. Liu, J. Hu, Appl. Catal. B, 2018, 234, 109-116.

[38] H. Zhao, L. Guo, C. Xing, H. Liu, X. Li, J. Mater. Chem. A, 2020, 8, 1955-1965.

[39] E. Nurlaela, S. Ould-Chikh, I. Llorens, J. L. Hazemann, K. Takanabe, Chem. Mater., 2015, 27, 5685-5694.

[40] M. Han, H. Wang, S. Zhao, L. Hu, H. Huang, Y. Liu, Inorg. Chem. Front., 2017, 4, 1691-1696.

[41] M. Khasu, T. Nyathi, D. J. Morgan, G. J. Hutchings, M. Claeys, N. Fischer, Catal. Sci. Technol, 2017, 7, 4806-4817.

[42] Y. Zhu, T. Wan, X. Wen, D. Chu, Y. Jiang, Appl. Catal. B, 2019, 244,
814-822.

[43] C. W. Kung, H. W. Chen, C. Y. Lin, K. C. Huang, R. Vittal, K. C. Ho, ACS Nano, 2012, 6, 7016-7025.

[44] J. Zhang, Z. Yu, Z. Gao, H. Ge, S. Zhao, C. Chen, S. Chen, X. Tong, M. Wang, Z. Zheng, Y. Qin, Angew. Chem. Int. Ed., 2017, 56, 816-820.

[45] B. H. Meekins, P. V. Kamat, ACS Nano, 2009, 3, 3437-3446.

[46] H. Yang, Z. Jin, G. Wang, D. Liu, K. Fan, Dalton Trans., 2018, 47, 6973-6985.

[47] W. Sun, X. Meng, C. Xu, J. Yang, X. Liang, Y. Dong, C. Dong, Y. Ding, Chin. J. Catal., 2020, 41, 1826-1836.

[48] D. Sun, J. W. Shi, D. Ma, Y. Zou, G. Sun, S. Mao, L. Sun, Y. Cheng, Chin. J. Catal., 2020, 41, 1421-1429.

[49] L. Qi, J. Yu, M. Jaroniec, Phys. Chem. Chem. Phys., 2011, 13, 8915-8923.

[50] J. Low, J. Yu, M. Jaroniec, S. Wageh, A. A. Al-Ghamdi, Adv. Mater., 2017, 29, 1601694.

[51] D. Huang, M. Wen, C. Zhou, Z. Li, M. Cheng, S. Chen, W. Xue, L. Lei, Y. Yang, W. Xiong, W. Wang, Appl. Catal. B, 2020, 267, 118651.

[52] R. Marschall, Adv. Funct. Mater., 2014, 24, 2421-2440.

\title{
表面组装钴物种同时促进 $\mathrm{Cd}_{0.9} \mathrm{Zn}_{0.1} \mathrm{~S}$ 光催化剂的界面电荷分离和表面反应
}

\author{
Khakemin Khan ${ }^{\mathrm{a}, \mathrm{b}}$, 徐丽粉 ${ }^{\mathrm{a}, \mathrm{b}}$, 石 明 ${ }^{\mathrm{a}, \mathrm{b}}$ ，曲江珊 ${ }^{\mathrm{a}, \mathrm{b}}$ ，陶晓萍 ${ }^{\mathrm{a}}$ ，冯兆池 ${ }^{\mathrm{a}}$ ，李 灿 ${ }^{\mathrm{a}}$ ，李仁贵 ${ }^{\mathrm{a},{ }^{*}}$ \\ ${ }^{\mathrm{a}}$ 中国科学院大连化学物理研究所, 催化基础国家重点实验室, 洁净能源国家实验室(筹), 辽宁大连116023 \\ 中 中国科学院大学, 北京 100049
}

\begin{abstract}
摘要: 利用人工光合成将太阳能转化为化学燃料是太阳能利用的重要途径, 具有广阔的应用前景, 其中, 太阳能光催化分 解水制氢是最为关键的反应之一. 但是, 大多数半导体光催化材料面临着光生电荷分离困难和表面催化反应速率慢等挑 战. 本文以具有可见光响应的半导体光催化剂 $\mathrm{Cd}_{0.9} \mathrm{Zn}_{0.1} \mathrm{~S}(\mathrm{CZS})$ 纳米棒为研究模型, 利用水热法成功在其表面上均匀地组装

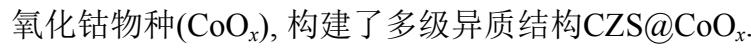

扫描电子显微镜和透射电子显微镜显示, 表面组装的 $\mathrm{CoO}_{x}$ 物种均匀地覆盖在CZS纳米棒的整个表面上, 形成了有序的 $\mathrm{CZS} @ \mathrm{CoO}_{x}$ 核壳多级异质结构. 高分辨率透射电子显微镜进一步确认了氧化钴晶格间距与六方 CZS 的 $(002)$ 晶面高度匹配,

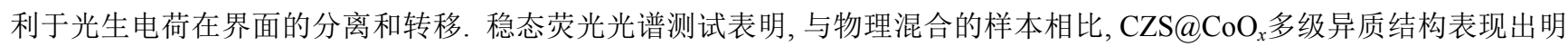
显降低的荧光强度, 说明多级异质结构能有效促进光生电子-空穴对的分离. 时间分辨荧光光谱结果显示, $\mathrm{CZS} @ \mathrm{CoO}_{x}$ 多级 异质结构的平均光生电荷寿命明显增长, 进一步确认了多级异质结构对光生电荷分离的作用. 此外, 电化学开路电位测量 显示, 增强的开路电压响应归因于多级异质结构CZS@CoO 中致密的界面接触. 电化学阻抗谱进一步确认, 与没有形成致 密界面结构的 $\mathrm{CZS}-\mathrm{CoO}_{x}$ 和 $\mathrm{CZS} / \mathrm{CoO}_{x}$ 相比, 多级异质结构 $\mathrm{CZS} @ \mathrm{CoO}_{x}$ 的电荷转移电阻大幅度降低, 从而确保了更快的界面 电荷分离和转移. 最后对CZS@ $\mathrm{CoO}_{x}$ 多级异质结构的光催化产氢活性进行了评价, 发现其光催化产氢的性能远高于贵金属 $\mathrm{Pt} / \mathrm{CZS}$ 光催化剂; 进一步测量了CZS@ $\mathrm{CoO}_{x}$ 的表观量子效率, 在 $420 \mathrm{~nm}$ 处光催化产氢的表观量子效率为 $20 \%$. 此外, 在多 级异质结构 $\mathrm{CZS} @ \mathrm{CoO}_{x}$ 上进一步引入Pt助催化剂, 可将表观量子效率进一步提升至 $37 \%$. 本文报道的这一简易可行的表面 组装构建多级异质结构的策略有望在太阳能光催化领域发挥重要作用.
\end{abstract}

关键词: 异质结构; 界面电荷分离; 表面反应; 光催化产氢

收稿日期: 2020-08-24. 接受日期: 2020-09-11. 上网日期: 2020-11-22.

*通讯联系人. 电话: (0411)84379698; 传真: ((0411)84694447; 电子信箱: rgli@dicp.ac.cn

基金来源: 国家自然科学基金(21633010, 21673230); 大连化学物理研究所创新基金(DICP I202031); 兴辽英才计划 (XLYC1907078).

本文的电子版全文由Elsevier出版社在ScienceDirect上出版(http://www.sciencedirect.com/science/journal/18722067). 